\title{
In situ monitoring of rolling-circle amplification on a solid support by surface plasmon resonance and optical waveguide spectroscopy
}

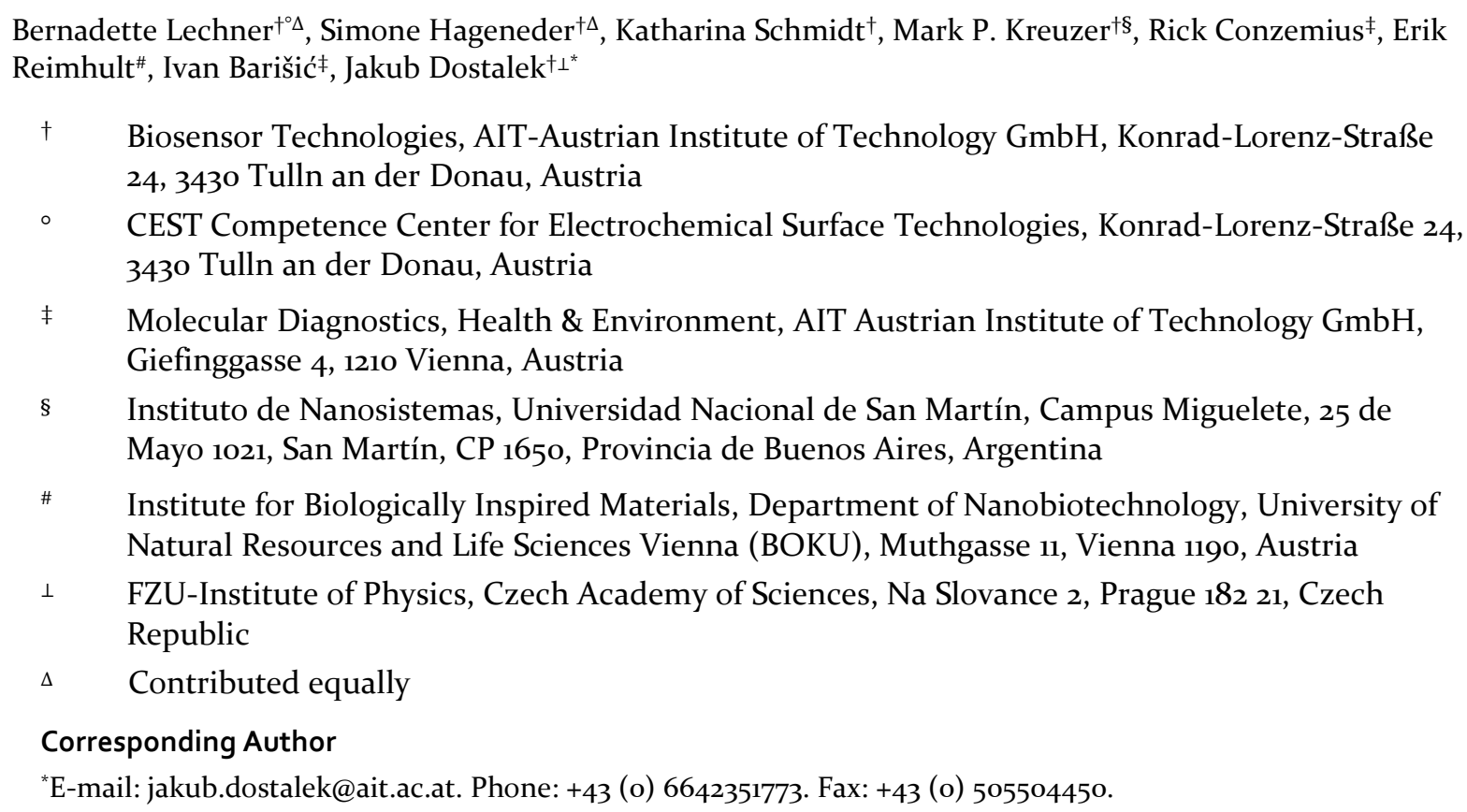

\section{Supporting software}

The optical instrument for SPR and SPFS measurements was supported by a dedicated software Wasplas (developed by Andreas Scheller at Max Planck Institute for Polymer Research, Mainz, Germany). The instrument was operated in the angular scan mode or in the kinetics mode. The angular scan mode enabled sweeping the angle of incidence $\theta$ and recording the angular reflectivity $R(\theta)$ and fluorescence $F(\theta)$ spectra for the subsequent analysis of guided SP and dielectric optical waveguide modes $\left(\mathrm{TEM}_{1,2, \ldots}\right)$ probing the interfaces. In the kinetics mode, the angle of incidence $\theta$ was set close to the minimum of SP resonance in the region where the slope $\mathrm{d} R / \mathrm{d} \theta$ was highest and a shift of the resonant coupling to SP to higher angle was associated with the increase in the reflectivity signal $R$. The recorded angular scans $R(\theta)$ were analyzed by a software Winspall (developed at Max Planck Institute for Polymer Research, Mainz, Germany). Based on the Fresnel reflectivity model, the non-linear regression was used for fitting the series of reflectivity dips in $R(\theta)$ associated with the resonant excitation of surface plasmon SP and dielectric optical waveguide $\mathrm{TEM}_{1,2, \ldots}$ modes in the TM or TE polarization. In the model, a stack of layers defined by thickness $d$ and refractive index $n$ was used and these parameters varied to fit the reflectivity curves. 


\section{Mathematical models}

In the following section we describe the mathematical model for the determination of surface mass density $\Gamma$ for immobilized layers on a plasmonic biosensor, carrying a mixed thiol-SAM. The data discussed in the section "In-situ monitoring of chain growth" (Fig. 4 and 5) is used as example to facilitate the understanding of the presented data and established models (see Table S1 and S2).

\section{Surface mass density $\Gamma$ :}

$$
\Gamma=d_{\mathrm{p}} \cdot\left(n_{\mathrm{p}}-n_{\mathrm{b}}\right) /(d \mathrm{n} / d \mathrm{c})
$$

where $d_{\mathrm{p}}$, is the thickness of the polymer layer, $n_{\mathrm{p}}$ is refractive index of the polymer layer, $n_{\mathrm{b}}$ is refractive index of the aqueous solution contacted with the polymer layer, and $d n / d c$ is a factor determined for biopolymers., ${ }^{1,2}$ The determined $\Gamma$ for each layer allows to determine the:

Grafting density $\sigma$ of immobilized protein and DNA molecules (neutravidin, biotin/TS-ICP):

$$
\sigma=\Gamma / M_{\mathrm{w}}
$$

Table S1: Determined thickness $d_{\mathrm{p}}$, surface mass density $\Gamma$ and grafting density $\sigma$ for the neutravidin and the target sequence padlock duplex (biotin/TS-|CP) layer, respectively. The refractive index of the aqueous solution in contact with the protein/DNA layer was PBST buffer $\left(n_{\mathrm{b}}=1.333\right)$.

\begin{tabular}{|l|c|c|c|c|c|c|}
\hline \multicolumn{1}{|c|}{ Layer } & $\begin{array}{c}d_{\mathrm{p}} \\
{[\mathrm{nm}]}\end{array}$ & $n_{\mathrm{p}}$ & $\begin{array}{c}(\mathrm{dn} / \mathrm{dc}) \\
{[\mathrm{mm} / \mathrm{mg}]}\end{array}$ & $\begin{array}{c}\Gamma \\
{\left[\mathrm{ng} / \mathrm{mm}^{2}\right]}\end{array}$ & $\begin{array}{c}M_{\mathrm{w}} \\
{[\mathrm{kDa}]}\end{array}$ & $\begin{array}{c}\sigma \\
{\left[\mathrm{nmol} / \mathrm{mm}^{2}\right]}\end{array}$ \\
\hline Neutravidin (protein) & 2.9 & \multirow{2}{*}{1.45} & 0.2 & 1.70 & 67 & $2.5 \cdot 10^{-5}$ \\
\hline biotin/TS-|CP duplex (DNA) & 1.6 & & 0.17 & 1.10 & 42.3 & $2.6 \cdot 10^{-5}$ \\
\hline
\end{tabular}

Average number of nucleotides per chain generated by RCA (Table $2 \mathrm{~S}$ ):

$$
\# \mathrm{nt}_{(\text {per chain })}=\Gamma_{(\mathrm{RCA})} / \sigma_{(\text {biotin/TS-|CP) }} / M_{\mathrm{w}(\text { padlock })} \# \mathrm{nt}_{(\text {padlock })}
$$

where $\Gamma_{(\mathrm{RCA})}$ is the surface mass density determined for the ssDNA adlayer formed by the RCA. The estimated mass per generated DNA chain $m_{\text {(chain) }}$ is obtained by assuming that each immobilized target|padlock DNA duplex (at a grafting density of $\sigma_{(\text {(biotin/TS-ICP) }}$ ) got prolonged. The molecular weight of the \#nt $\left(\right.$ padlock) $=81$ nucleotide long padlock probe is $M_{\text {w(padlock) }}=25 \mathrm{kDa}$.

Table S2: Values for thickness $d_{\mathrm{p}}$ and refractive index $n_{\mathrm{p}}$ of the grown DNA brushes at 240 minutes of RCA with

\begin{tabular}{|c|c|c|c|c|c|c|c|c|c|c|c|}
\hline $\begin{array}{l}\text { Model to } \\
\text { fit } R(\theta)\end{array}$ & $\left.\begin{array}{c}d_{\mathrm{p} 1} \\
{[\mu \mathrm{m}]}\end{array}\right]$ & $n_{\mathrm{p} 1}$ & $\begin{array}{c}d_{\mathrm{p} 2} \\
{[\mu \mathrm{m}]}\end{array}$ & $n_{\mathrm{p} 2}$ & $\left.\begin{array}{c}d_{\mathrm{p} 3} \\
{[\mu \mathrm{m}]}\end{array}\right]$ & $n_{\mathrm{p} 3}$ & $\begin{array}{c}d_{\mathrm{p}(1+2+3)} \\
{[\mu \mathrm{m}]}\end{array}$ & $\begin{array}{c}\text { weighted } \\
n_{\mathrm{p}(1+2+3)}\end{array}$ & $\begin{array}{c}\Gamma_{\mathrm{RCA}(\mathrm{t}=240 \mathrm{~min})} \\
{\left[\mathrm{ng} / \mathrm{mm}^{2}\right]}\end{array}$ & $\begin{array}{l}m_{\text {(chain) }} \\
{[\mathrm{MDa}]}\end{array}$ & $\begin{array}{c}\text { \#nt per chain } \\
{[\mathrm{kb}]}\end{array}$ \\
\hline one-box & 11.2 & 1.3423 & - & - & - & - & 11.2 & 1.3423 & 415.1 & 15.9 & 51.7 \\
\hline two-box & 3.2 & 1.3440 & 9.1 & 1.3406 & - & - & 12.3 & 1.3415 & 396.8 & 15.2 & 49.4 \\
\hline three-box & 3.8 & 1.3434 & 6.0 & 1.3410 & 2.9 & 1.3384 & 12.7 & 1.3411 & 382.8 & 14.7 & 47.6 \\
\hline
\end{tabular}
the established one-three-box model. The polymer brushes were in contact with an aqueous environment with a refractive index $n_{\mathrm{b}}=1.336$.

The determined values can then be used to assess further characteristics as e.g. polymer volume content as well as the influence of different ionic environments on the biointerface containing densely packed DNA polymer brushes. 
Average extension rate of $\Phi_{29}$-Polymerase:

Assuming that the average number of nucleotides is $\sim 52 \mathrm{~kb}$ per chain at a RCA reaction time of 240 minutes, the average extension rate is $\sim 215 \mathrm{nt} / \mathrm{min}$.

Contour length of RCA product:

$$
L=\# \mathrm{nt}_{(\text {per chain) }} \cdot a
$$

where $a$ is the effective monomer (nucleotide) length and value from literature $a=0.6$ nm was used [3].

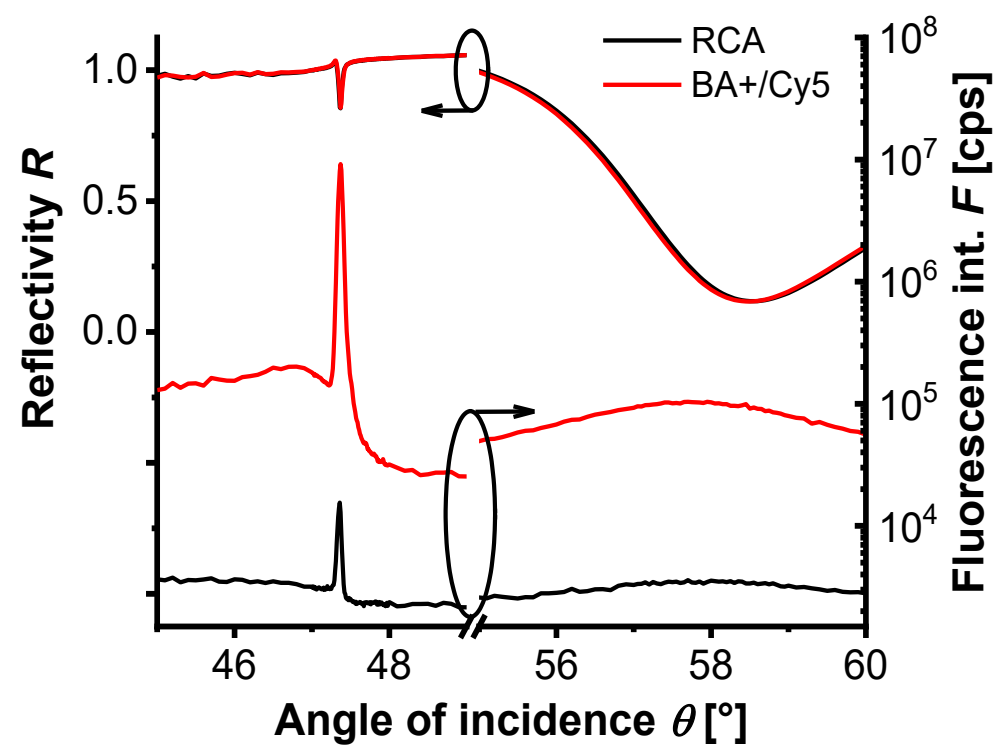

Figure S1: Measured angular reflectivity scan $R(\theta)$ and angular fluorescence intensity scan $\mathrm{F}(\theta)$ for) RCA brush reacted with BA/Cy5 in PBST.

\section{References}

(1) Tumolo, T.; Angnes, L.; Baptista, M. S. Determination of the Refractive Index Increment (Dn/Dc) of Molecule and Macromolecule Solutions by Surface Plasmon Resonance. Anal. Biochem. 2004, 333 (2), 273-279. https://doi.org/10.1016/j.ab.2004.06.010.

(2) Theisen, A; Johann, C; Deacon, M. P; Harding, S. . Refractive Increment Data-Book for Polymer and Biomolecular Scientists; Nottingham University Press: Nottingham, 2000.

(3) Chi, Q.; Wang, G.; Jiang, J. The Persistence Length and Length per Base of Single-Stranded DNA Obtained from Fluorescence Correlation Spectroscopy Measurements Using Mean Field Theory. Phys. A Stat. Mech. its Appl. 2013, 392 (5), 1072-1079.

https://doi.org/10.1016/j.physa.2012.09.022. 
